\title{
A New Relative Method of Polymer Molecular Weight Determination
}

\author{
Lech GMACHOWSKI \\ Institute of Physical Chemistry, Polish Academy of Sciences, \\ Kasprzaka 44/52, 01-224 Warsaw, Poland
}

(Received August 15, 1985)

\begin{abstract}
A new relative method of polymer weight determination is developed on the basis of the previously [Polym. J., 18, 783 (1986)] elaborated permeable sphere model. According to this method, the molecular weight of a given polymer may be determined by comparison of its sedimentation coefficient measured at a concentration in the dilute regime with the sedimentation coefficient of the same polymer of known molecular weight in the same solvent. The comparison of sedimentation coefficients must be performed at concentrations corresponding to the same values of the packing fraction of coils. The new method is verified for sedimentation coefficients measured for both a polymer-solvent system at the theta temperature and for a polymer-good solvent system.

KEY WORDS Brinkman's Model / Determination of Polymer Molecular Weight / Semidilute Polymer Solution / Sedimentation Coefficient /
\end{abstract}

\section{THEORY}

In a previous paper, ${ }^{1}$ a model was presented for the fluid flow relative to a swarm of identical permeable spheres, and it proved to give a good description of the concentration dependence of sedimentation coefficient in both dilute and semidilute regimes and for different polymer-solvent systems. This model predicts an interdependence of three dimensionless parameters $\lambda R, P R$, and $\delta^{3}$

$$
f\left(\lambda R, P R, \delta^{3}\right)=0
$$

where $R$ is the coil radius, $\lambda$ and $P$ are the reciprocal square roots of the solution permeability and coil permeability, respectively, and $\delta^{3}$ is the volume fraction occupied by coils.

In the semidilute region these three parameters $\left(\lambda R, P R\right.$, and $\left.\delta^{3}\right)$ are constant and their values depend on the exponent $n$ in the empirical equation

$$
\lambda=\text { const }_{1}(1-\varepsilon)^{n}
$$

which connects the $\lambda$-value in this region with the solution porosity $\varepsilon$. The $P R$-parameter is assumed to remain constant over the whole range of both semidilute and dilute solutions, whereas the volume fraction occupied by coils $\delta^{3}$ in the dilute regime is proportional to the packing fraction of coils $\xi$ defined by

$$
\xi=\frac{1-\varepsilon}{1-\varepsilon^{*}}
$$

according to the following equations

$$
\begin{aligned}
\delta_{\text {dilute }}^{3} & =\frac{1-\varepsilon}{1-\varepsilon_{\text {coil }}}=\frac{1-\varepsilon^{*}}{1-\varepsilon_{\text {coil }}} \cdot \frac{1-\varepsilon}{1-\varepsilon^{*}} \\
& =\delta_{\text {semidilute }}^{3} \cdot \xi=\text { const }_{2} \cdot \xi
\end{aligned}
$$

where $\varepsilon^{*}$ is the porosity at the transition between the two domains of concentration.

As a consequence of the above considerations, eq 1 may be simplified to the form valid in the dilute regime:

$$
\lambda R=f(\xi)
$$

The form of $f(\xi)$ depends on the exponent $n$ in eq 2 and should be the same for all polymersolvent systems with the same $n$-value. In particular, this function for a given polymer- 
solvent system, is independent of polymer molecular weight. The polymer molecular weight is represented ${ }^{1}$ by

$$
M=\text { const }_{3} R^{3}\left(1-\varepsilon_{\text {coil }}\right)
$$

For a constant $\xi$ one obtains from eq 4

$$
\left(1-\varepsilon_{\text {coil }}\right) \sim(1-\varepsilon)
$$

and from eq 5

$$
R \sim 1 / \lambda
$$

so eq 6 may be rearranged into the following proportionality

$$
M \sim(1-\varepsilon) / \lambda^{3}
$$

By taking into account the definition of $\lambda$ for a sedimenting system ${ }^{1}$

$$
\lambda=\sqrt{\frac{\left(1-\rho_{1} / \rho_{\mathrm{s}}\right) c}{\mu \mathrm{s}}}
$$

where $c=\rho_{\mathrm{s}}(1-\varepsilon), \rho_{1}$ and $\rho_{\mathrm{s}}$ are the solvent density and solute density, respectively, and $\mu$ is the solvent viscosity, the proportionality of eq 9 may be expressed in terms of the commonly used quantities of sedimentation coefficient $s$ and concentration $c$ or solute weight fraction $w$, which is nearly propirtional to $c$, as follows:

$$
M \sim s^{1.5} c^{-0.5} \quad \text { for } \quad \xi=\text { const }
$$

To use this proportionality, the equation describing lines of constant $\xi$ must be known. For a constant $\xi$ the following equations may be derived from eq 8,7 , and 6 , respectively

$$
\begin{aligned}
& \frac{\mathrm{d} \ln \lambda}{\mathrm{d} \ln (1-\varepsilon)}=-\frac{\mathrm{d} \ln R}{\mathrm{~d} \ln (1-\varepsilon)} \\
& \frac{\mathrm{d} \ln R}{\mathrm{~d} \ln (1-\varepsilon)}=\frac{\mathrm{d} \ln R}{\mathrm{~d} \ln \left(1-\varepsilon_{\text {coil }}\right)} \\
& \frac{\mathrm{d} \ln M}{\mathrm{~d} \ln \left(1-\varepsilon_{\text {coil }}\right)}=1+3 \frac{\mathrm{d} \ln R}{\mathrm{~d} \ln \left(1-\varepsilon_{\text {coil }}\right)}
\end{aligned}
$$

From eq 11-13 the following relationship may be derived: $\left.\frac{\mathrm{d} \ln \lambda}{\mathrm{d} \ln (1-\varepsilon)}\right|_{\xi=\text { const }}=\frac{1}{3}\left[1-\frac{\mathrm{d} \ln M}{\mathrm{~d} \ln \left(1-\varepsilon_{\text {coil }}\right)}\right]$

The value of the right side of eq 14 is independent of $\xi$ and thus must be equal to its value for $\xi=1$. Hence, eq 14 reduces by eq 2 to

$$
\frac{\mathrm{d} \ln \lambda}{\mathrm{d} \ln (1-\varepsilon)}=n
$$

By means of eq $11-15$, it can be shown that in the $\log \lambda$ vs. $\log (1-\varepsilon)$ plot lines of constant $\xi$ are straight and parallel to the line described be eq 2 .

Practical application of eq 10 may be made in the following way. On a log-log plot one represents sedimentation coefficient measured over a large concentration interval, for a given polymer of known molecular weight $M_{\mathrm{s}}$ as a function of concentration. In the semidilute regime the line should be straight. By measuring at a low concentration the sedimentation coefficient for the same polymer of the molecular weight $M_{\mathrm{x}}$ to be determined, one get the point $\left(c_{\mathrm{x}}, s_{\mathrm{x}}\right)$ in the plot. Through the point $\left(c_{\mathrm{x}}, s_{\mathrm{x}}\right)$ one draws the line parallel to the straight line, obtaining the point of intersection $\left(c_{\mathrm{s}}, s_{\mathrm{s}}\right)$ with the $s(c)$-reference curve. The molecular weight $M_{\mathrm{x}}$ then can be calculated according to the following equation:

$$
M_{\mathrm{x}}=\left(\frac{s_{\mathrm{x}}}{s_{\mathrm{s}}}\right)^{1.5}\left(\frac{c_{\mathrm{x}}}{c_{\mathrm{s}}}\right)^{-0.5} M_{\mathrm{s}}
$$

\section{VERIFICATION}

Previously ${ }^{1}$ it was concluded on the basis of experimental data ${ }^{2}$ that for $0.03<\xi<0.1$, the molecular weight of a given polymer calculated from the model is higher than a nominal one, but their ratio is a unique function of $\xi$ for different nominal molecular weights considered. For $\xi<0.1$ it is thus possible to apply the new relative method described in this paper. Experimental data ${ }^{2}$ verify in this way the method for $\xi<0.1$. As an additional veri- 


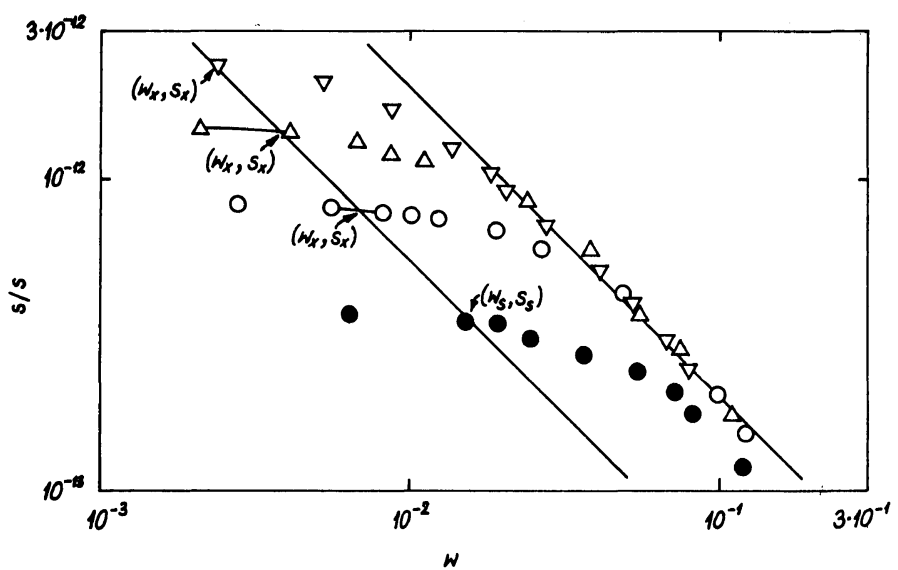

Figure 1. The new relative method of determining molecular weight of polystyrene using sedimentation data in cyclopentane: $\bigcirc, M_{\text {nominal }}=2.04 \times 10^{4} ; \bigcirc, M_{\text {nominal }}=1.1 \times 10^{5} ; \triangle, M_{\text {nominal }}=3.9 \times 10^{5} ; \nabla$, $M_{\text {nominal }}=9.5 \times 10^{5}$.

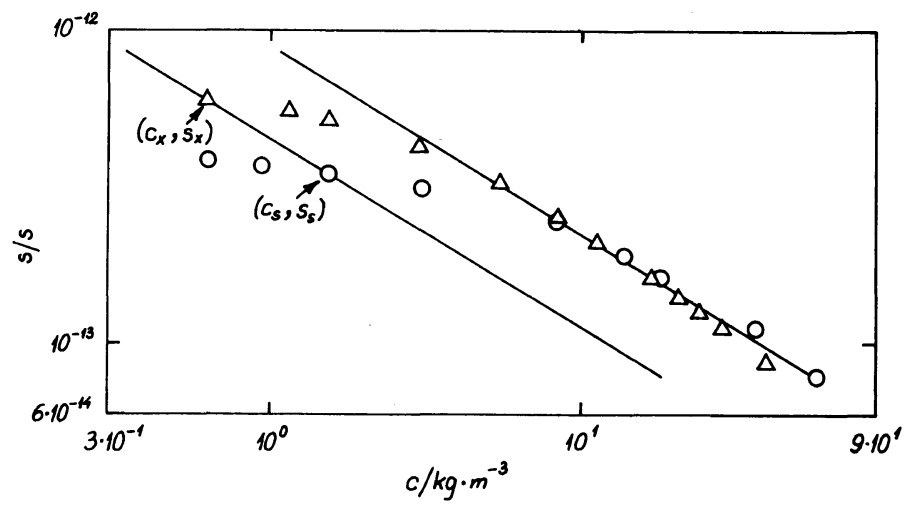

Figure 2. The new relative method of determining molecular weight of polystyrene using sedimentation data in toluene: $\bigcirc, M_{\text {nominal }}=1.1 \times 10^{5} ; \triangle, M_{\text {nominal }}=3.9 \times 10^{5}$.

fication presented, data of sedimentation coefficient are employed, for which the absolute method cannot be used.

Many experimental data are available for sedimentation coefficients but they are usually not corrected for pressure effects to obtain values valid for atmospheric pressure. However, since the experiments were performed under closely identical conditions, the sedimentation coefficients determined correspond to a certain average pressure. Such sedimentation coefficient values are regarded to be proportional to the values for atmospheric pressure. ${ }^{3}$ So the relative variation of sedi-

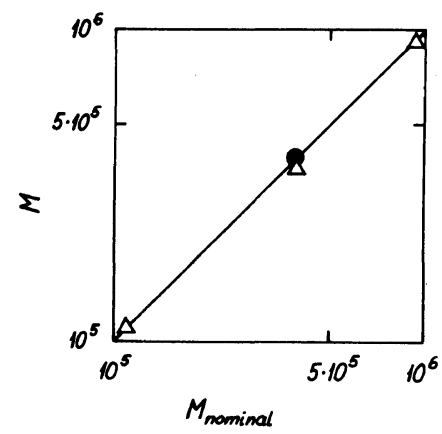

Figure 3. Plot of $M$ vs. $M_{\text {nominal }}$ for the systems indicated: $\triangle$, polystyrene in cyclopentane; $\boldsymbol{O}$, polystyrene in toluene. 
mentation coefficient with concentration is retained. Equation 10 should be thus exact for such data.

The additional verification is performed for two polymer-solvent systems, one at the theta temperature and the other in a good solvent.

In the $\log s v s . \log c$ plot, a line parallel to the straight line valid in the semidilute regime should intersect lines in a dilute regime at points for which eq 16 is valid. The molecular weight will be calculated by taking the lowest $M_{\text {nominal }}$ as a reference.

The first system is polystyrene in cyclopentane at the theta temperature, for which sedimentation coefficients were measured by Nyström et al. ${ }^{4}$ over a large concentration interval. Nominal molecular weights were $2.04 \times 10^{4}, 1.1 \times 10^{5}, 3.9 \times 10^{5}$, and $9.5 \times 10^{5}$.

The second system is polystyrene of nominal molecular weights of $1.1 \times 10^{5}$ and $3.9 \times 10^{5}$ in toluene at $25^{\circ} \mathrm{C}$, for which sedimentation coefficients were measured by Nyström et al. ${ }^{5}$

Experimental data and the constant $\xi$-lines are shown in Figures 1 and 2.

The molecular weights calculated by eq 16 are compared in Figures 3 with their nominal values.

A good agreement may be observed for both systems analyzed.

\section{REFERENCES}

1. L. Gmachowski, Polym. J., 18, 783 (1986).

2. P. F. Mijnlieff and W. J. M. Jaspers, Trans. Faraday Soc., 67, 1837 (1971).

3. B. Nyström and L.-O. Sundelöf, Chemica Scripta, 10, 16 (1976).

4. B. Nyström, J. Roots, and R. Bergman, Polymer, 20, 157 (1979).

5. B. Nyström, B. Porsch, and L.-O. Sundelöf, Eur. Polym. J., 13, 683 (1977). 